\title{
Synthetic MR Imaging Sequence in Daily Clinical Practice
}

W e read with great interest the article published in June by Granberg et al, "Clinical Feasibility of Synthetic MRI in Multiple Sclerosis: A Diagnostic and Volumetric Validation Study." ${ }^{1}$ At the moment, another technique called MR fingerprinting allows quantitative $\mathrm{T} 1, \mathrm{~T} 2$, and proton density measurements (and potentially other parameters such as diffusion) and has been presented at most MR imaging international meetings as very promising. Indeed, MR imaging quantification (through the MR fingerprinting method) was clearly expected to be "more accurate and reproducible than traditional MR imaging" by the European Society of Radiology. ${ }^{2}$ However, its use in clinical daily practice is, at the moment, not consistently evaluated. Synthetic MR imaging is yielding identical outcomes for radiologists, which is, in our opinion, an enormous advantage. This is a more readily available technique which already gives us access to these quantified parameters on a daily basis thus allowing us to evaluate this future evolution of MR in today's clinical practice. In addition, synthetic MR imaging has the ability to produce morphologic conventional sequences, particularly in diseases such as multiple sclerosis, with considerable time-savings. Other clinical applications of this sequence, in our opinion, will be in oncologic patients and the assessment of hydrocephaly and the syndrome of the trephined, ${ }^{3}$ due to its ability to efficiently and reliably quantify lesions in the brain parenchyma and also to detect increased or decreased volume of CSF.

Further developments are necessary, nevertheless, until its use in clinical routine and replacement of conventional sequences can be a reality. For instance, technical developments are needed, as

http://dx.doi.org/10.3174/ajnr.A4895 mentioned in the article, to solve problems related to artifacts such as partial volume effects in FLAIR sequences, which may mimic a subarachnoid hemorrhage (this effect was also reported with MR fingerprinting methodology) (Fig 1). ${ }^{4}$

Finally, with the technical improvements likely to take place, we think this sequence could be applied to other organ systems for selected pathologies and that the related findings will be essential for the evolution to systematic quantitative MR imaging.

\section{REFERENCES}

1. Granberg T, Uppman M, Hashim F, et al. Clinical feasibility of synthetic MRI in multiple sclerosis: a diagnostic and volumetric validation study. AJNR Am J Neuroradiol 2016;37:1023-29 CrossRef Medline

2. European Society of Radiology (ESR). Magnetic resonance fingerprinting: a promising new approach to obtain standardized imaging biomarkers from MRI. Insights Imaging 2015;6:163-65 CrossRef Medline

3. Vasung L, Hamard M, Soto MC, et al. Radiological signs of the syndrome of the trephined. Neuroradiology 2016;58:557-68 CrossRef Medline

4. Deshmane A, McGivney D, Badve C, et al. Accurate synthetic FLAIR images using partial volume corrected MR fingerprinting. In: Proceedings of the Annual Meeting and Exhibition of International Society for Magnetic Resonance in Medicine; Singapore. May 7-13, 2016; Poster No. 1909 


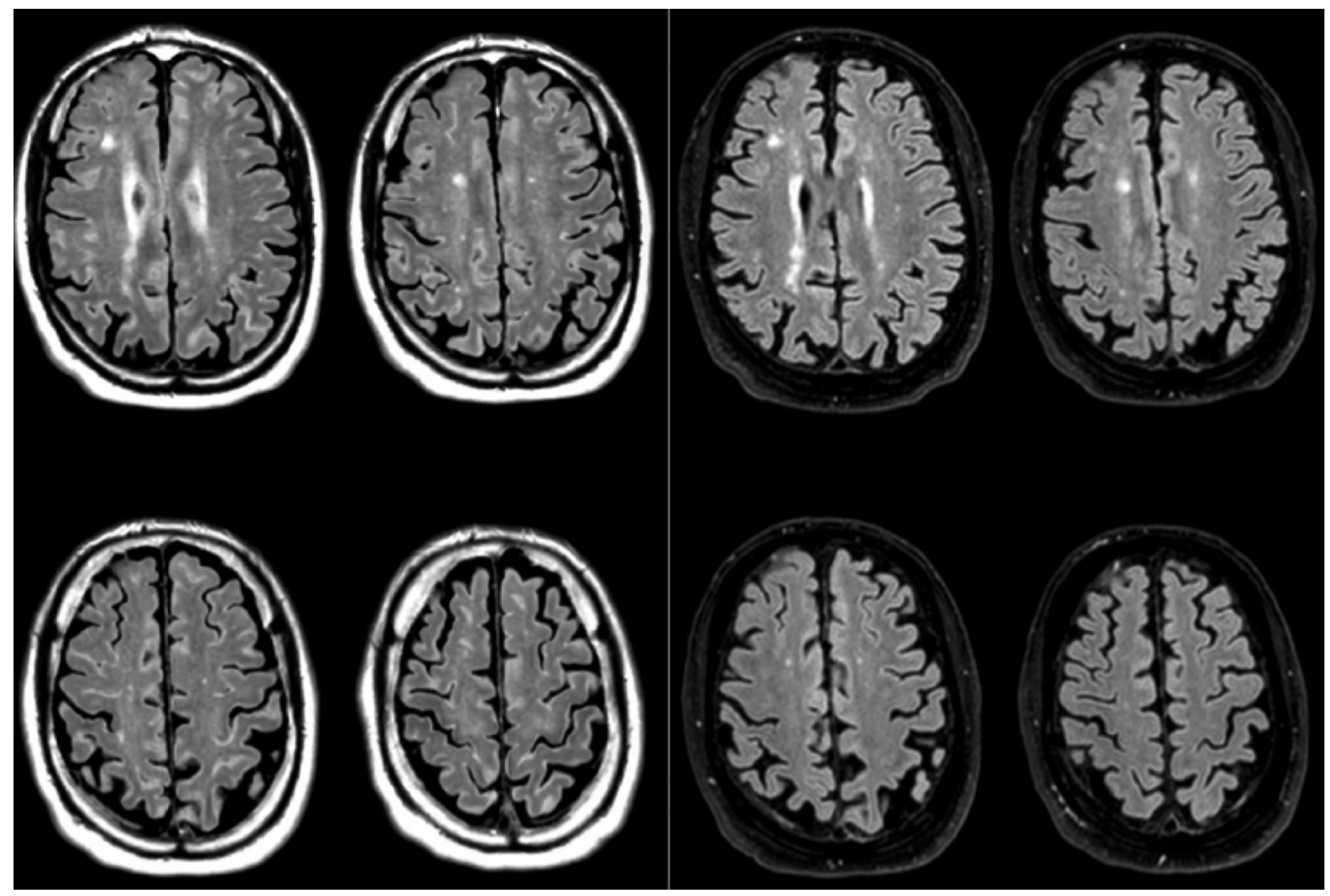

FIG 1. Four consecutive sections acquired with a synthetic MR imaging T2 FLAIR sequence (left) and a conventional FLAIR sequence (right) in the same patient at the same time. Note hyperintensities within and adjacent to the cerebral cortex on the synthetic sequence mimicking a subarachnoid hemorrhage. These abnormalities disappear on the conventional FLAIR sequence. 